\title{
Reticulocyte Hemoglobin Distribution Width
}

National Cancer Institute

\section{Source}

National Cancer Institute. Reticulocyte Hemoglobin Distribution Width. NCI Thesaurus.

Code C139070.

A measurement of the distribution of the hemoglobin concentration in reticulocytes. 